\section{Response to Discussion of "Formation of an Intermediate Layer Between Grains in Nickel-Based Superalloy Turbine Blades"}

\author{
KEEHYUN KIM and PAUL WITHEY
}

DOI: $10.1007 / \mathrm{s} 11661-017-4256-\mathrm{x}$

(C) The Author(s) 2017. This article is an open access publication

The authors would like to thank Professor Campbell for his interest in the paper, "Formation of an Intermediate Layer Between Grains in Nickel Based Superalloy Turbine Blades," published in the June edition of Metallurgical and Materials Transactions $\mathrm{A}^{[1]}$ and also our thanks go to the editors of the journal for allowing a response to be made to the points raised.

Professor Campbell's work on oxide bi-films in castings is well known and the authors understand the implications for castings in general and nickel based superalloys in particular. In the case of the grain boundary defects in the aforementioned paper the authors feel that the grain defects are not caused by these bi-films and their arguments can be categorized into two groups.

Firstly there is no evidence of the oxide film in any of the sections of grain boundaries investigated. Professor Campbell identifies Figure 8(b) in the paper as giving indications of the bi-film, however, this is contrast due to the edge effect in the SEM near the interface and most of all, it was confirmed at high magnification and resolution that there was no oxide. In addition, as the assumed thickness of $20 \mathrm{~nm}$ for an oxide film is well within the capability of the microscopes used (see References 2 and 3), and if this layer existed there would be some evidence of this film in the sections viewed.

Transmission electron microscopy element maps taken proximate to each side of the grain boundary (Figure 1) clearly show that there was no oxide layer detected at either the non-coherent (sharp) boundary or the coherent side of the boundary. As shown in this and a previous paper ${ }^{[4]}$ the non-coherent boundary between the bulk of the grain and the intermediate layer is sharp without any precipitates or voids. This sharp interface has also been confirmed in many other samples and with grain defects across a range of causes both thermal and solutal such as other stray grains, multigrain regions, freckle chain grains, and even low angle grain boundary. ${ }^{[5]}$ Therefore, it is apparent that bi-films themselves did not exist in the samples examined in this and other studies.

In other (unpublished) work the authors have also had occasion to investigate cracks in this alloy which were exacerbated through the presence of damaging elemental species. In this case the aluminum in the alloy was removed from the base material to form alumina particles along the sides of the crack. When these samples were ion beam milled the alumina, being much harder, remained proud of the surface (Figure 2). As such it would be expected that any alumina layer within the samples investigated would have also been easily identifiable after sample preparation for the TEM analysis through this morphological difference.

Secondly the proposed formation mechanism for the oxide films does not account for the microstructures observed, in particular, the presence of the rhenium rich phases on the coherent grain interface of the elongated $\gamma^{\prime}$ precipitates (Figure 3). Professor Campbell explains that the rhenium rich particles would, quite logically, 

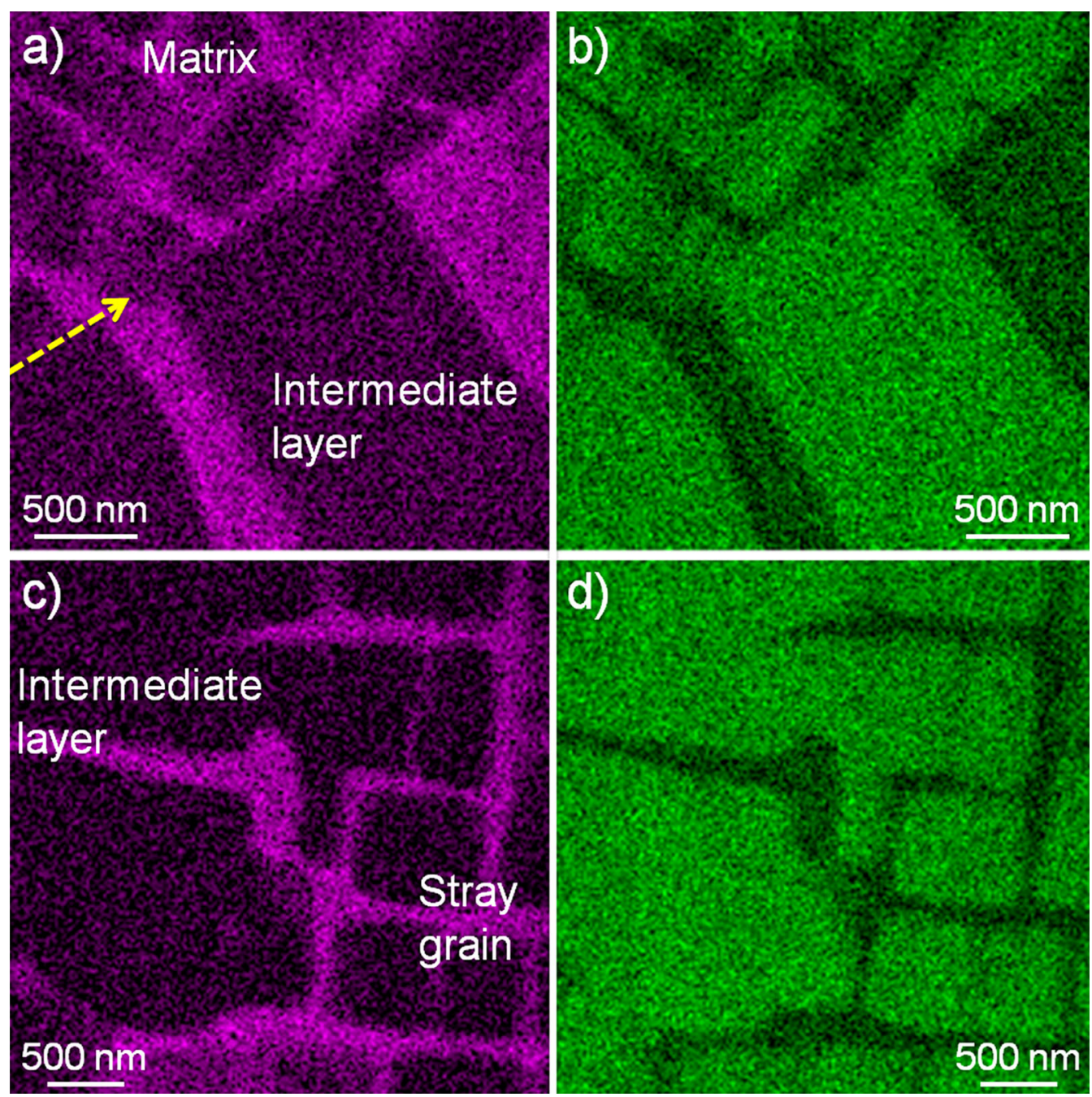

Fig. 1-STEM-EDX element maps of $\operatorname{Re}(a, c)$ and $\mathrm{Al}(b, d)$ near the boundary of an intermediate layer and a matrix (a, b) and near the boundary of intermediate/stray grain (c, d). Note that the arrow in panel (a) indicates the sharp boundary. ${ }^{[4]}$

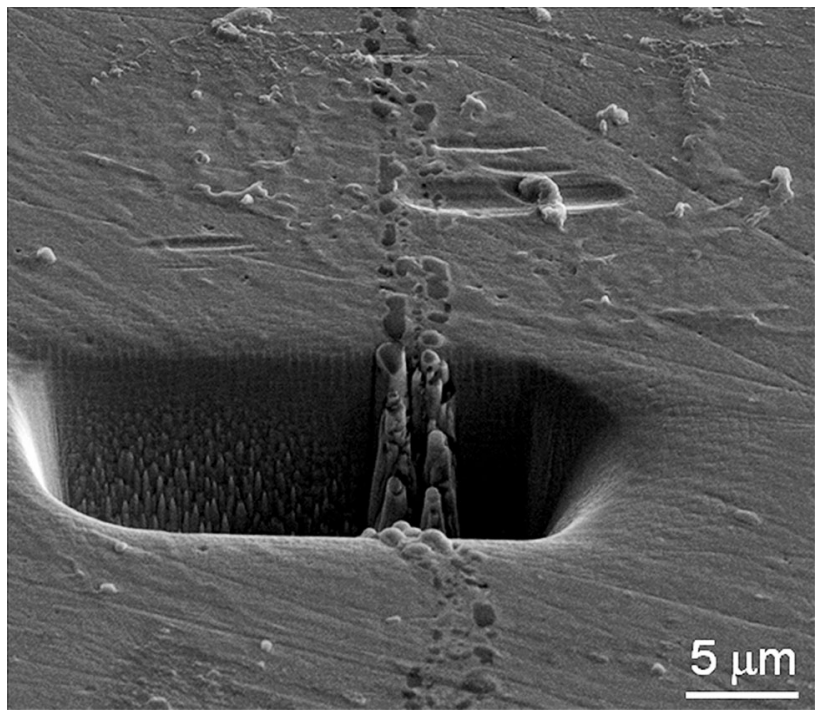

Fig. 2-Sample showing the difference in ion beam response of alumina in a third generation superalloy. be found at grain boundaries having been driven there during solidification. However, the explanation that there is an alumina film preventing the solidification progressing further and hence co-located with the grain boundary and rhenium particles, is not supported by the evidence of the elongated $\gamma^{\prime}$ which extends beyond the rhenium particles by 4 to $5 \mu \mathrm{m}$. In reality the $\gamma^{\prime}$ does not exist during the initial stage of solidification and the peak temperature during solution heat treatment as this is above the $\gamma^{\prime}$ solvus. If there was an alumina layer present on the boundary then the $\gamma^{\prime}$ would not extend through it but would be restricted by it, and the alumina layer would be at the non-coherent interface (Figure 4). If the grain boundary is thought to be at the non-coherent interface then the rhenium rich particles come to rest $4 \mu \mathrm{m}$ from the boundary with the alloy still in the molten state.

Given the discussion above, the authors feel that the grain defects observed could not be caused by oxide bi-films. This is through the lack of evidence for the presence of the films and also because the mechanism 

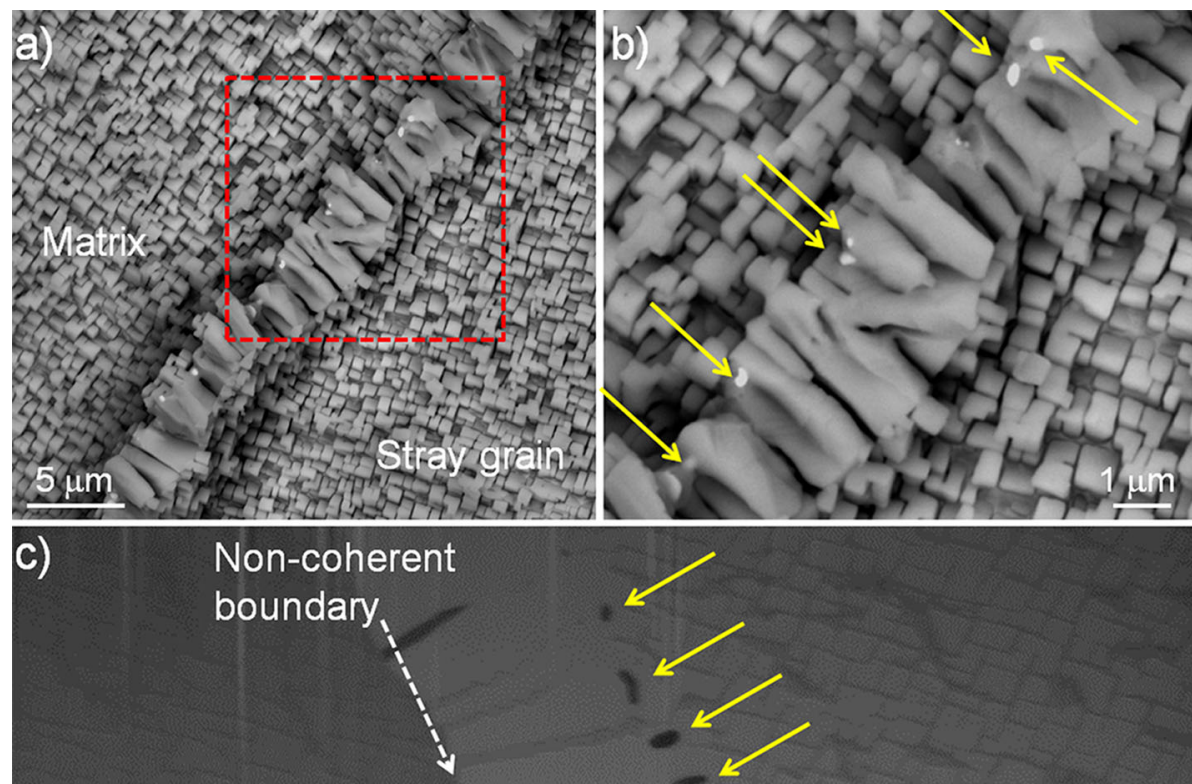

Stray grain

Matrix
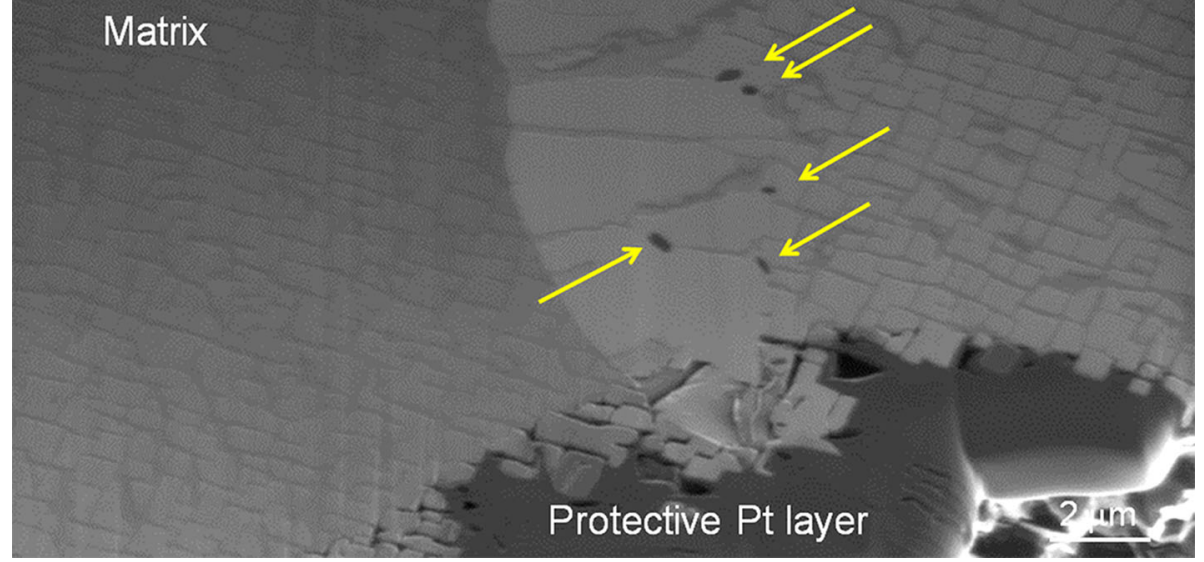

Fig. 3-SEM images near the intermediate layer: $(a, b)$ top view (a) and magnified image in (a) (b), and (c) tilting-view image after cross-sectioning by FIB. The arrows in (b) and (c) indicate Re-rich particles.
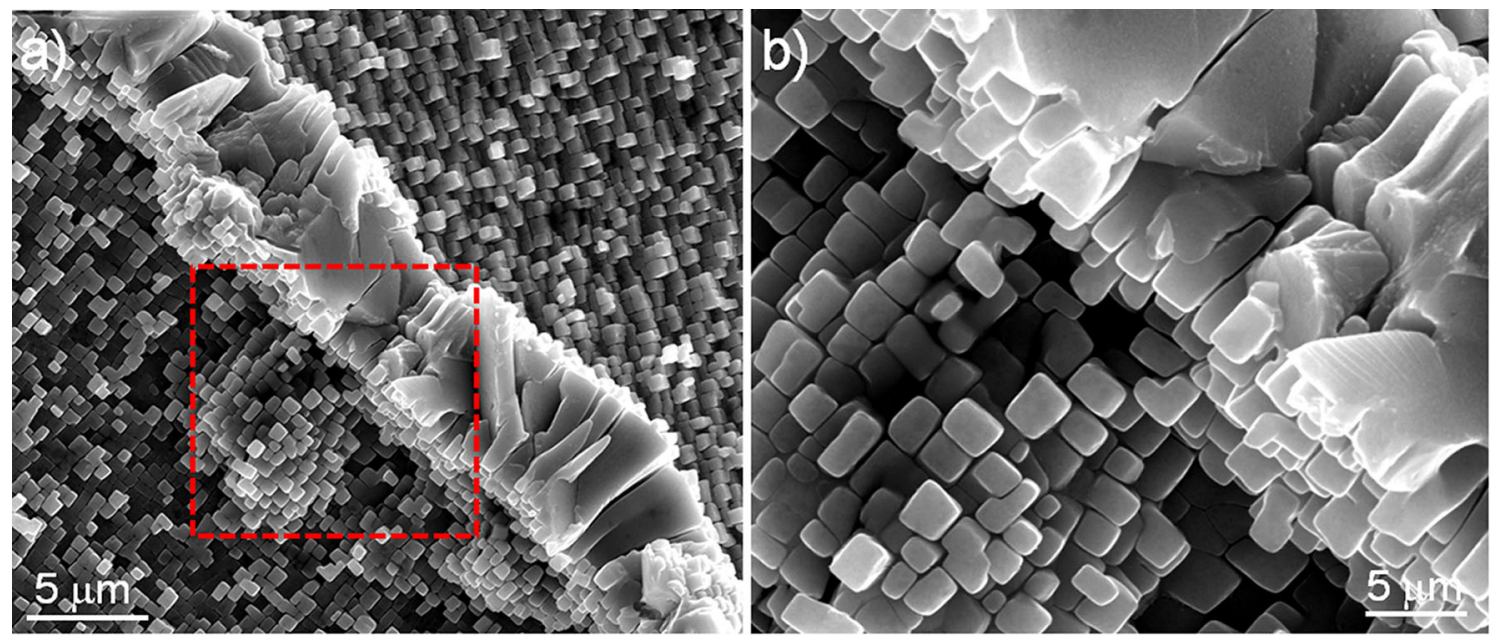

Fig. 4-SEM images showing elongated $\gamma^{\prime}$ phases which formed from $\gamma^{\prime}$ phases in a matrix grain. 
proposed by Professor Campbell is not supported by the observations made on the grain boundary regions.

\section{OPEN ACCESS}

This article is distributed under the terms of the Creative Commons Attribution 4.0 International License (http://creativecommons.org/licenses/by/4.0/), which permits unrestricted use, distribution, and reproduction in any medium, provided you give appropriate credit to the original author(s) and the source, provide a link to the Creative Commons license, and indicate if changes were made.

\section{REFERENCES}

1. K. Kim and P.A. Withey: Metall. Mater. Trans. A, 2017, vol. 48A, pp. $2932-42$.

2. K. Kim and S. Kuroda: Scripta Mater., 2010, vol. 63, pp. 215-18.

3. K Kim, M Watanabe, K Mitsuishi, K Lakoubovskii, and S Kuroda: J. Phys. D: Appl. Phys., 2009, vol. 42, p. 065304.

4. K. Kim, P.A. Withey, and W.D. Griffiths: Metall. Mater. Trans. A, 2015, vol. 46A, pp. 1024-29.

5. K. Kim and P.A. Withey: Mater. Trans., 2016, vol. 57, pp. $1698-1706$. 